Revista Brasileira de Informática na Educação - RBIE Brazilian Journal of Computers in Education (ISSN online: 2317-6121; print: 1414-5685)

http://br-ie.org/pub/index.php/rbie

$\begin{array}{llll}\text { Submission: 20/04/2020; } & 1^{\text {st }} \text { round notif.: 27/08/2020; } & \text { New version: 13/10/2020; } & 2^{\text {nd }} \text { round notif.: 07/12/2020; } \\ \text { Camera ready: 11/12/2020; } & \text { Edition review: 14/12/2020; } & \text { Available online: 20/12/2020; } & \text { Published: 20/12/2020; }\end{array}$

\title{
Habilidades para Vida e Tecnologias Digitais Educacionais: Uma Revisão Sistemática de Literatura
}

\author{
Title: Life Skills and Digital Educational Technologies: A Systematic Literature Review
}

\author{
Fabiana Maris Versuti \\ USPRP \\ fabiana_versuti@usp.br
}

Carmem Beatriz Neufeld

USPRP

cbneufeld@usp.br

\author{
Juliana de Lima \\ USPRP \\ juliana.delima@usp.br
}

\author{
Isabela Pizarro Rebessi \\ USPRP \\ isabela.rebessi@usp.br
}

\begin{abstract}
Resumo
Pesquisas relacionadas à temática das Habilidades para Vida vem sendo tema de investigações científicas que têm evidenciado e esclarecido como se dão os processos comportamentais e socioemocionais relacionados com dimensões essenciais para êxito ao longo da vida, tais como atendimento as demandas acadêmicas, sociais e profissionais, relacionamentos interpessoais, entre outros. Tomando-se como referência o ambiente escolar, um dos pontos chave para o desenvolvimento destas habilidades, este estudo visa sintetizar as evidências disponíveis no panorama da literatura científica brasileira acerca de intervenções focados em desenvolvimento de Habilidades para Vida, que utilizem Tecnologias Digitais Educacionais como ferramenta interventiva, nos últimos dez anos. Para isto, foi feito uma revisão sistemática de literatura, que compreendeu etapas subsequentes e encadeadas, em formato baseado no modelo da Plataforma Evidências - Módulo Sumarize (SUMARIZE), que foi utilizado para as etapas de buscas e de redação deste artigo. O SUMARIZE foi utilizado para orientar o desenvolvimento de protocolos de revisões sistemáticas que ajudaram os autores a melhorarem o relato de revisões sistemáticas e meta-análises, como é o escopo desta pesquisa. Os principais resultados concentram-se em dez artigos que abarcaram de modo articulado os dois construtos, Habilidades para Vida e tecnologias digitais educacionais. Em linhas gerais, evidencia-se que o ambiente escolar é um espaço subaproveitado para inserção das tecnologias educacionais como mediadora de acesso à aprendizagem, com grande potencial para o trabalho com Habilidades para Vida.
\end{abstract}

Palavras-Chave: Revisão Sistemática; Tecnologias Digitais Educacionais, Habilidades para Vida

\begin{abstract}
Research related to the theme of Life Skills has been the subject of scientific investigations that have highlighted and clarified how the behavioral and socioemotional processes take place related to essential dimensions for success throughout life, such as attending academic, social and professional demands, interpersonal relationship skills, among others. Taking the school environment as a reference, one of the key points for the development of these skills, this study aims to synthesize the available evidence in the panorama of the Brazilian scientific literature about interventions focused on the development of Life Skills, which use Educational Digital Technologies as an intervention tool, in the last ten years. For this, a systematic literature review was carried out, which included subsequent and linked stages, in a format based on the Evidences Platform model - Summarize Module (SUMARIZE), which was used for the search and writing stages of this article. SUMARIZE was used to guide the development of systematic review protocols that helped authors improve the reporting of systematic reviews and meta-analyzes, as is the scope of this research. The main results are concentrated in ten articles that comprehensively encompassed the two constructs, Life Skills and educational digital technologies. In general, it is evident that the school environment

Cite as: Versuti, F. M., Neufeld, C. B., de Lima, J., \& Rebessi, I. P. (2020). Life Skills and Digital Educational Technologies: A Systematic Literature Review (Habilidades para Vida e Tecnologias Digitais Educacionais: Uma Revisão Sistemática de Literatura). Brazilian Journal of Computers in Education (Revista Brasileira de Informática na Educação - RBIE), 28, 1105-1119. DOI: 10.5753/RBIE.2020.28.0.1105
\end{abstract}


is an underused space for the insertion of educational digital technologies as a mediator of access to learning, with great potential for working with Life Skills.

Keywords: Systematic Review; Educational Technologies, Life Skills

\section{Introdução}

A proliferação do debate a respeito das demandas educacionais do mundo contemporâneo e do discurso a favor do desenvolvimento integral necessário ao indivíduo do século XXI, reportam à diversas áreas do conhecimento, dentre elas a Psicologia, Educação, Tecnologias Digitais aplicadas à Educação. Especificamente, neste cenário de "efervescência" discursiva, a presente pesquisa se insere, buscando evidências de intervenções realizadas em escolas, que focam em Habilidades para Vida por intermédio de tecnologias digitais educacionais, a partir de uma revisão sistemática da literatura.

As Habilidades para Vida tem sido tema de investigações educacionais recentes com resultados relevantes acerca de processos essenciais para êxito acadêmico do aluno. Evidências apontam para a sua importância ao longo de todo desenvolvimento sendo necessário um bom repertório orientado pelos princípios da Competência Social, que se referem à mediação entre pensamento, sentimentos e comportamentos que o um indivíduo deve ser capaz de fazer, levando em consideração seu contexto ambiental e cultural (Nardi, Ferreira, \& Neufeld, 2017), e que são subsidiadas pelas habilidades cognitivas e pelo seu amadurecimento, uma vez que é isto que possibilita refinamentos que futuramente subsidiarão todas as habilidades listadas no campo das Habilidade para Vida. Por habilidades cognitivas considera-se que a cognição é um conjunto de funcionalidades mentais que incluem a atenção, concentração, percepção, compreensão, memória, resolução de problemas e raciocínio, que são habilidades que permitem aos indivíduos o pleno exercício de sua cidadania (Ramos, Lorenset, \& Petri, 2016).

Para este estudo, faz-se necessário contextualizar que o referencial de Habilidades para Vida adotado baseia-se no documento publicado pela Organização Mundial da Saúde que afirma que tais habilidades viabilizam a aprendizagem de demais habilidades, permitindo o enfrentamento saudável de possíveis situações de risco à saúde física e mental, oriundas de conflitos nos mais diversos contextos relacionais, como família, pares, parceiros amorosos e professores (WHO, 1997; Murta et al., 2009). De forma que as Habilidades para Vida são habilidades que promovem o desenvolvimento de comportamentos adaptativos para os indivíduos lidarem de maneira mais positiva com as demandas da vida cotidiana, sendo elas: resolução de problemas, pensamento criativo, pensamento crítico, empatia, tomada de decisão, comunicação eficaz, relacionamento interpessoal, autoconhecimento, manejo de emoções e manejo de estresse (WHO, 1997).

Embora o construto das habilidades cognitivas, como já apresentado no início do texto, esteja associado a outros conceitos importantes na literatura, como a aprendizagem, resolução de problemas, levantamento de hipóteses, planejamento das ações e raciocínio, entre outras; ao abordar-se a concepção de Habilidades para Vida $(\mathrm{HV})^{1}$, assume-se um construto integrador desses aspectos ao abarcar o desenvolvimento de habilidades emocionais, sociais e cognitivas que auxiliam o indivíduo a ser competente - de acordo com o conceito apresentado de Competência Social - no manejo das demandas cotidianas, sendo este último o conceito adotado por esta pesquisa (Nardi et al., 2017).

Neste cenário, a Organização Panamericana de Saúde, via Organização Mundial de Saúde, destaca que a escola é um dos locais mais adequados para intervenções de promoção e prevenção de saúde, tanto pelo fato de as crianças e adolescentes passarem grande parte de seu dia no

\footnotetext{
${ }^{1}$ Para simplificar a leitura deste artigo a sigla HV foi adotada em substituição ao termo habilidades para vida.
} 
ambiente escolar, quanto pelo importante papel da escola junto à sociedade (WHO, 2010). Logo, a escola é palco privilegiado para o aprendizado das Habilidades para Vida contribuindo para o desenvolvimento o integral dos alunos (Paiva \& Rodrigues, 2008).

O artigo elaborado por Costa, Duqueviz, e Pedroza (2015) aborda que um mediador de acesso à aprendizagem, e que com grande potencial para o trabalho com Habilidades para Vida, são as Tecnologias Digitais e da Informação e Comunicação (TDIC). Porém, há entraves para o uso dessa mediação, relacionadas com a natureza do sistema de socialização dos jovens de hoje que é essencialmente diferente da maneira como as gerações anteriores se comunicam, tal fato cria um abismo entre a forma como professor tenta se conectar com seus alunos e a forma como estes estudantes recebem estas informações.

Outros estudos que se dedicaram a analisar o perfil dos estudantes que atualmente cursam o Ensino Básico os caracterizam como hipertecnológicos, pois desde seu nascimento estão inseridos em um ambiente social imerso no uso dos recursos digitais. Porém, as instituições escolares frequentemente não acompanham a velocidade deste processo de inovação e apresentam dificuldades para inserir as tecnologias adotadas pelos jovens no cotidiano escolar, fazendo com a que escola se torne um universo quase à parte para grande parcela de seu público-alvo (Bezarra, Lima, Brito, \& Santos, 2019).

Para Bastos e Siqueira (2020) essa nova realidade traz alterações na forma como os estudantes aprendem, uma vez que ganham e perdem foco com extrema facilidade, dificilmente conseguindo manter-se concentrado em apenas uma atividade por um longo período de tempo. O que pode, erroneamente, dar a impressão de que são capazes de fazer múltiplas tarefas ao mesmo tempo, porém as pesquisas indicam que a alternância entre tarefas proporcionadas pela tecnologia tem trazido para esta geração grande dificuldade em manter o foco. Para os autores, se torna crucial ao ambiente escolar conseguir garantir a aprendizagem destes jovens de forma mais rápida e eficiente, por meio da ação e observação, do desenvolvimento de espaços mais criativos e motivadores, que perpassam também por uma reestruturação de novas práticas pedagógicas como por exemplo, estímulo às atividades em grupos, exploração de diferentes linguagens, especialmente os relacionados ao uso das tecnologias digitais.

A realidade escolar contemporânea também evidencia a necessidade de o foco do ensino ser para além do domínio dos conteúdos disciplinares, a demanda é por um ensino integrado, que contemple as $\mathrm{HV}$, uma vez que são estas que efetivamente comportam o desenvolvimento das habilidades cognitivas, que dizem respeito ao uso de variadas funções mentais. Portanto, pode-se entender este desenvolvimento como o meio responsável por tornar os indivíduos competentes em interagir simbolicamente com o ambiente, uma vez que este desenvolvimento está associado a capacidade que os estudantes terão de monitorar seus pensamentos, sentimentos, ideias e intenções, para então comunicá-los e de forma reflexiva conseguir identificar esses mesmos processos nos outros. Desta forma, intervenções neste ambiente voltadas para o desenvolvimento integral do estudante devem considerar a inserção dos indivíduos para além da escola, levando-se em consideração ambientes como familiar, social e futuramente o ambiente de trabalho, construindo saberes científicos em conjunto com o desenvolvimento dos recursos cognitivos como por exemplo reflexão e o pensamento crítico, que são bases necessárias para os indivíduos exercerem habilidades de autocuidado, ou controle inibitório que futuramente servirá como arcabouço preventivo para evitação de comportamentos de risco (Minto, Pedro, Netto, Bugliani, \& Gorayeb, 2006; Ramos et al., 2016; Sargiani \& Maluf, 2018; Bezarra et al., 2019).

Uma vez que as intervenções em HV têm como objetivo o desenvolvimento de estratégias para um enfrentamento saudável das demandas do dia-a-dia, como manejo de emoções e estresse, empatia, autoconhecimento e habilidades de comunicação, os programas envolvendo a criação dessas competências podem ocorrer em diversos contextos, inclusive e preferencialmente nas escolas (Neufeld, Daolio, Cassiano, Rossetto, \& Cavenage, 2014). 
Sendo assim, em 2004 a OMS passou a indicar como desejável e recomendável que programas de promoção e prevenção de saúde incluíssem as Habilidades para Vida (WHO, 2004), e desde então a literatura vem apontando bons resultados advindos de programas de HV, como demonstrado pelos autores Minto, Pedro, Netto, Bugliani e Gorayeb (2006) ao realizarem um grupo de ensino de HV na escola para adolescentes. As sessões eram de duas horas e foram realizados 12 encontros com frequência semanal. Foram trabalhados temas permeados por 10 Habilidades para Vida, como capacidades pessoais, habilidades e limites; diferenças pessoais, preconceitos e estereótipos; maneiras de lidar com emoções desagradáveis; reconhecimento dos sentimentos; comunicação não-verbal; busca de alternativas para resolução de problemas; dicas para uma boa comunicação, entre outros.

Sendo reconhecida no documento formulado pela Unesco, em 2015: Educação para Cidadania Global - preparando alunos para os desafios do século XXI, a capacidade que a Educação tem de compor a solução dos problemas globais atuais nas dimensões sociais, políticas, culturais, econômicas e ambientais, não limitando seu papel ao de apenas ensinar conteúdos pedagógicos, mas de também ser ferramenta de transmissão e construção de valores, atitudes e de promover transformação social, por meio das Habilidades para Vida (UNESCO, 2015). E neste sentido, o relatório anterior da UNESCO traz a recomendação para o uso das novas tecnologias educacionais como uma ferramenta que viabiliza um desenvolvimento dos processos de ensino e de aprendizagem, evidenciando o papel articulador da tecnologia no cenário educacional (Delors et al., 1998).

Uma vez que pesquisas vêm desempenhando um papel cada vez mais central no desenvolvimento de Políticas Públicas, como mostra Hypólito (2011), se faz amplamente necessário e urgente conhecer o que o panorama científico brasileiro apresentou na última década de pesquisas tanto no que se referem às propostas, quanto no que se referem às preocupações, achados e hipóteses para a conjuntura educacional no Brasil, visando agregar evidências de pesquisa que possam contribuir com esta construção social.

Nesse sentido, se dá importância de se discutir a utilização das Tecnologias Digitais da Informação e Comunicação (TDIC's) como instrumentos mediadores da aprendizagem. Uma vez que para a área da Educação é fundamental conseguir acompanhar o desenvolvimento tecnológico para poder se apropriar dessas ferramentas e conseguir atuar de forma apropriada com habilidades que visem a formação de um sujeito saudável nos âmbitos social, pessoal, intelectual, emocional e físico. Para isso, este estudo tem como objetivo sintetizar as evidências disponíveis no panorama da literatura científica brasileira de intervenções focadas no desenvolvimento de HV, que utilizem Tecnologias Digitais Educacionais como ferramenta interventiva e que tenham sido publicadas nos últimos dez anos.

\section{$2 \quad$ Método}

A revisão sistemática da literatura aqui proposta compreende etapas subsequentes e encadeadas, em um formato que se inspira no modelo do Plataforma Evidências - Módulo Sumarize (SUMMARIZE, disponível em https://qeed.nees.com.br/), utilizada para as buscas e redação do artigo oriundo da presente revisão sistemática. Participaram da seleção e avaliação do estudo três avaliadoras independentes, presentes em todas as etapas do processo.

As etapas são descritas a seguir:

I. Definição colegiada feita por especialistas sobre os descritores, critérios de inclusão/exclusão e bases de dados;

II. Busca pelos estudos em vários tipos de documentos; 
III. Triagem dos estudos face aos critérios de inclusão;

IV. Síntese e mapeamento dos trabalhos;

V. Definição de uma agenda futura de pesquisa.

A string inicial oferecido pela plataforma QeeD foi ("Aluno" OR "Alunos" OR "Estudante" OR "Estudantes" OR "Student") AND ("Habilidades de Vida" OR "Habilidades para Vida " OR "Life skills" OR "Programas Educacionais" OR "Educational policies " OR "Educational programs" OR "Políticas educacionais" OR "Tecnologias Educacionais" OR "Educational Digital Technologies" OR "Educational Technologies " OR "TDIC's" OR "Tecnologias digitais da educação") AND ("Educação" OR "Children Education" OR "Educacional" OR "Education" OR "Educação Básica" OR "Escola" OR "Sala de aula"). De acordo com as bases de dados e os estudos encontrados, a string sofreu alterações na organização das palavras e nos operadores booleanos.

\subsection{Seleção dos Artigos}

A etapa 1 consistiu em uma pré-seleção por análise de títulos: com base nos critérios definidos pelo colegiado de especialistas (Tabela 1) a primeira etapa de seleção de artigos foi baseada nos títulos encontrados nas bases científicas Scielo, IEE, EduBase, Google Scholar, Science Direct e Scopus. A figura 1 aponta o número de artigos selecionados e excluídos em cada uma das etapas. Inicialmente foram incluídos 101 artigos na plataforma Qeed e, após o processo de seleção, foram incluídos 10 estudos na presente revisão. A etapa 2 consistiu na leitura dos resumos dos artigos selecionados, enquanto na etapa 3 houve uma expansão dos construtos pesquisados por conta do baixo número inicial de artigos encontrados. Por fim, na etapa 4, foi feita a análise dos dados dos artigos selecionados por meio da plataforma Sumarize.

Tabela 1: Critérios de inclusão e exclusão.

\begin{tabular}{|c|c|}
\hline \multicolumn{2}{|c|}{ Critérios de Seleção } \\
\hline Critérios de Inclusão & Critérios de Exclusão \\
\hline $\begin{array}{c}\text { A metodologia de pesquisa na qual a } \\
\text { literatura se baseia deve ser explicitada } \\
\text { (tamanho da amostra, instrumentos, } \\
\text { procedimento de coleta de dados) }\end{array}$ & Trabalhos duplicados \\
\hline $\begin{array}{c}\text { A literatura deve contemplar os anos } \\
\text { condizentes à Educação Básica }\end{array}$ & $\begin{array}{c}\text { Trabalhos que não estejam em } \\
\text { língua inglesa ou portuguesa }\end{array}$ \\
\hline $\begin{array}{c}\text { A literatura deve estar diretamente } \\
\text { relacionada a uma das três questões } \\
\text { (habilidades para vida, programas } \\
\text { educacionais ou tecnologias } \\
\text { educacionais) }\end{array}$ & $\begin{array}{c}\text { Trabalhos que não contemplem } \\
\text { integralmente a temática }\end{array}$ \\
\hline $\begin{array}{c}\text { Publicações feitas entre os anos de 2009 } \\
\text { e 2019 }\end{array}$ & $\begin{array}{c}\text { Publicações anteriores ao ano } \\
\text { de 2019 }\end{array}$ \\
\hline $\begin{array}{c}\text { Necessariamente os artigos devem } \\
\text { conter resumo }\end{array}$ & $\begin{array}{c}\text { Texto completo não disponível } \\
\text { gratuitamente }\end{array}$ \\
\hline
\end{tabular}


Os trabalhos devem ser conduzidos em território nacional
Trabalhos conduzidos em

território internacional


Figura 1. Fluxograma do processo de seleção e inclusão dos artigos.

\section{$3 \quad$ Análise de Dados}

A string sugerida pela plataforma Sumarize gerou zero resultados em todas as bases de dados selecionadas, sendo necessário fazer combinações entre as palavras-chave elencadas (Tabela 2) para gerar os resultados. Os dados encontrados foram filtrados a partir dos títulos conforme adesão ao escopo da pesquisa, considerando citações diretas das palavras-chave ou seus sinônimos ou referências indiretas ao escopo da pesquisa.

Tabela 2: Palavras-chave e sinônimos gerados pela plataforma Sumarize. 


\begin{tabular}{|c|c|}
\hline Palavra-Chave & Sinônimo \\
\hline Aluno & Alunos \\
& Criança \\
& Estudante \\
& Estudantes \\
& Student \\
\hline Educação & Children Education \\
& Educational \\
& Education \\
& Educação Básica \\
& Escola \\
& Sala de Aula \\
\hline Habilidades de Vida & Life Skills \\
& Habilidades para Vida \\
\hline Programas Educacionais & Educational Program \\
\hline Tecnologias Educacionais & Digital Educational Technologies \\
& TIDIC'S \\
\hline & Tecnologias Digitais da Educação \\
\hline
\end{tabular}

Após a primeira fase de seleção por títulos, os artigos passaram por uma leitura de seus resumos para checagem da metodologia utilizada; análise de consistência entre objetivos, metodologia utilizada e resultados encontrados; e pertinência ao tema no sentido de abarcarem tanto habilidades para vida, quanto o uso das tecnologias educacionais. Muitos artigos traziam populações alvo específicas que não eram aderentes à esta pesquisa, como por exemplo: população de mães lactantes em processo de aprendizagem sobre a importância da amamentação exclusiva, programas centrados na figura do professor para o trabalho e habilidades de vida e ensino de utilização de tecnologias educacionais digitais, entre outros, desta foram descartados por serem considerados fora do escopo desta pesquisa, mesmo quando apresentavam alguma das palavras-chave ou sinônimos do pool em seu título, resultando em apenas 2 artigos integralmente dentro do escopo determinado.

Dado o baixo número de artigos encontrados que apresentassem total adesão ao escopo desta pesquisa, foi detectada a necessidade de expandir a classificação dos construtos escolhidos, de forma a considerar outras dimensões presentes na inter-relação entre Habilidades para Vida e habilidades cognitivas. Esta escolha foi baseada no arcabouço teórico de Gorayeb (2002), Minto e colaboradores (2006) e Morales, Benitez e Santos (2013) que afirmam que as Habilidades para Vida são habilidades psicossociais que facilitam os processos de aprendizagem dos indivíduos. Assim sendo, a fase 3 de análise retomou o filtro de buscas anterior e expandiu a seleção dos artigos, neste momento considerando também habilidades cognitivas necessárias para $o$ desenvolvimento da leitura, escrita e letramento digital. Desta forma, os resultados da pesquisa foram mais amplos e conseguiram mapear da melhor forma o tema proposto, resultando em 101 artigos encontrados (Figura 1). 


\section{Artigos selecionados}

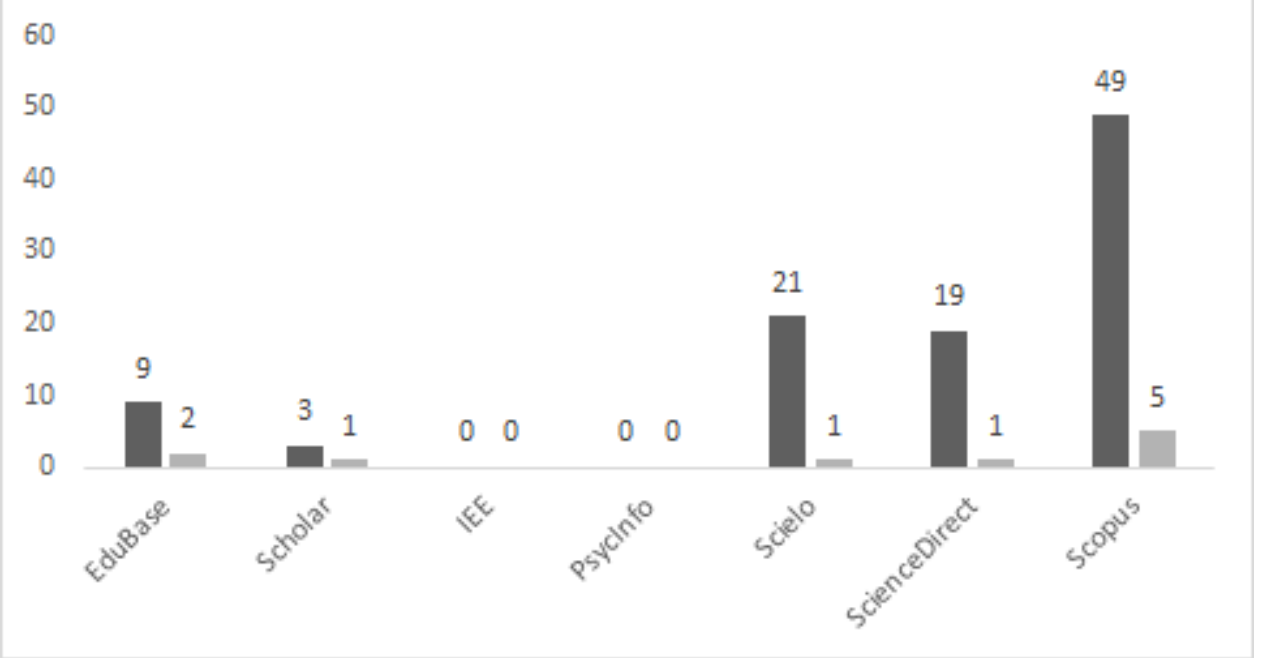

Figura 2: Artigos selecionados e aceitos por base de dados.

A última etapa, de análise de qualidade, foi concluída dentro da plataforma Sumarize e contemplou os momentos de definição dos critérios de qualidade (Tabela 3), notas referentes a cada critério, definição de nota de corte e input de todos os artigos encontrados desde a fase de busca inicial. Após este input e com os critérios da análise de qualidade definidos, a plataforma Sumarize viabiliza o momento de classificação dos artigos conforme os critérios estipulados. Por meio dos filtros estabelecidos foi possível encontrar 10 artigos compatíveis com o escopo estabelecido por esta pesquisa (Tabela 4).

Tabela 3: Critérios de qualidade e pontuação da plataforma Sumarize.

\begin{tabular}{|c|c|c|c|}
\hline \multirow[t]{2}{*}{ Critérios } & \multicolumn{3}{|c|}{ Pontuação } \\
\hline & Sim & $\begin{array}{c}\text { Atende } \\
\text { Parcialmente }\end{array}$ & Não \\
\hline $\begin{array}{l}\text { A amostra deve ser bem definida e representativa dos resultados } \\
\text { alcançados }\end{array}$ & 1.0 & 0.0 & 0.0 \\
\hline $\begin{array}{l}\text { O artigo traz políticas ou programas educacionais relacionados com } \\
\text { Habilidades de Vida }\end{array}$ & 1.0 & 0.5 & 0.0 \\
\hline $\begin{array}{l}\text { Os instrumentos aplicados na metodologia devem ser condizentes com os } \\
\text { resultados apresentados }\end{array}$ & 1.0 & 0.0 & 0.0 \\
\hline As intervenções devem ser bem fundamentadas na literatura & 1.0 & 0.0 & 0.0 \\
\hline Os resultados devem responder os objetivos apresentados & 1.0 & 0.5 & 0.0 \\
\hline A discussão deve ser bem fundamentada na literatura & 1.0 & 0.5 & 0.0 \\
\hline $\begin{array}{c}\text { São mencionadas as ameaças à validade e como estas ameaças afetam os } \\
\text { resultados e conclusões }\end{array}$ & 1.0 & 0.0 & 0.0 \\
\hline Houve a descrição ou referência a estudos simulares & 1.0 & 0.0 & 0.0 \\
\hline $\begin{array}{l}\text { O artigo informa o cronograma de execução, bem como tempo e/ou } \\
\text { período utilizado para realizar a coleta de dados }\end{array}$ & 1.0 & 0.0 & 0.0 \\
\hline
\end{tabular}




\begin{tabular}{|c|c|c|c|}
\hline Há uso de tecnologias educacionais ou digitais & 1.0 & 0.0 & 0.0 \\
\hline O público-alvo é referente ao escopo desta pesquisa & 1.0 & 0.0 & 0.0 \\
\hline $\begin{array}{c}\text { As conclusões são cabíveis dentro do alcance do estudo e dos resultados } \\
\text { apresentados }\end{array}$ & 1.0 & 0.0 & 0.0 \\
\hline \multirow[t]{2}{*}{ Score } & $\begin{array}{l}\text { Max } \\
\text { Score }\end{array}$ & & $\begin{array}{l}\text { Cutoff } \\
\text { Score }\end{array}$ \\
\hline & 12.0 & & 4.0 \\
\hline
\end{tabular}

Tabela 4: Fluxograma do processo de seleção dos artigos.

\begin{tabular}{|c|c|c|}
\hline & Artigos Elencados & Artigos excluídos \\
\hline Identificação & $\begin{array}{l}\text { Número de trabalhos } \\
\text { identificados nas bases: } 101\end{array}$ & $\begin{array}{c}\text { Artigos excluídos: } 50 \\
\text { Justificativa: artigos que não contemplam } \\
\text { integralmente o escopo da pesquisa }\end{array}$ \\
\hline Mapeamento & $\begin{array}{c}\text { Artigos triados, separados } \\
\text { para leitura de título e } \\
\text { resumo: } 51\end{array}$ & $\begin{array}{l}\text { Artigos excluídos após leitura de título/resumo: } 35 \\
\text { Justificativa: artigos não cumprem a priori os } \\
\text { critérios de inclusão ou eram duplicados }\end{array}$ \\
\hline Seleção & Artigos elegíveis: 16 & $\begin{array}{c}\text { Artigos excluídos: } 6 \\
\text { Justificativa: artigos não cumprem a priori os } \\
\text { critérios de inclusão }\end{array}$ \\
\hline Inclusão & Artigos incluídos: 10 & \\
\hline
\end{tabular}

Desta forma os artigos finais, com suas respectivas análises de qualidade seguem apresentados abaixo (Tabela 5).

Tabela 5: Artigos finais.

\begin{tabular}{|l|l|}
\hline Artigos Selecionados & \\
\hline $\begin{array}{l}\text { Can we teach to think in primary schools? A comparative analysis of the English and the } \\
\text { Brazilian National Curriculum and the impact of a small-scale cognitive enhancement study in } \\
\text { Brazil }\end{array}$ & $\begin{array}{l}\text { Matriz de referência para medição do alfabetismo nos domínios do letramento e do } \\
\text { numeramento }\end{array}$ \\
\hline $\begin{array}{l}\text { A Interação com jogos digitais e aprimoramento do controle inibitório: um estudo com crianças } \\
\text { do atendimento educacional especializado }\end{array}$ & \\
\hline Khan Academy: tecnologia favorável à aprendizagem matemática & \\
\hline
\end{tabular}




\begin{tabular}{|l|l|}
\hline Learning strategies mediated by technologies: use and observation of teachers & \\
\hline Letramento digital: impactos das tecnologias na aprendizagem da geração Y & \\
\hline Psicologia da educação e as tecnologias digitais de informação e comunicação & \\
\hline Studying in 3D environments & \\
\hline A promoção do uso de estratégias cognitivas em alunos do ensino médio \\
\hline $\begin{array}{l}\text { Aprendizagem criativa na construção de jogos digitais: uma proposta educativa no ensino de } \\
\text { ciências para crianças }\end{array}$
\end{tabular}

\section{Discussão}

Este estudo objetivou por meio de uma revisão sistemática da literatura, sintetizar as evidências disponíveis no panorama da literatura científica brasileira de intervenções focadas no desenvolvimento de Habilidades para Vida, que utilizaram Tecnologias Digitais Educacionais como ferramenta interventiva, publicadas nos últimos dez anos. Com base na análise dos artigos foram encontrados dez artigos que abarcaram de modo articulado os dois construtos: habilidades para vida e tecnologias digitais educacionais, a partir de referenciais teóricos que definam a temática e não apenas tratem de forma superficial o assunto.

Vale destacar que entre os estudos encontrados apenas dois especificamente contemplaram completamente os construtos das Habilidades para Vida e Tecnologias Digitais Educacionais (Marques, 2014; Sobreira, Viveiro, \& D'Abreu, 2018). Os demais artigos não possuem ênfase nas Habilidades para Vida, apesar de citá-las e relacioná-las com questões educacionais. Neste sentido, os achados desta revisão revelam uma preocupação elementar dos estudos em sistematizar quais estratégias seriam efetivas para construir práticas escolares condizentes com uma Educação para Cidadania Global (UNESCO, 2015), a partir da inserção de tecnologias digitais educacionais como ferramentas para a promoção de uma educação integral, de modo a preparar os estudantes para os desafios do século XXI e não limitando o papel da escolas ao de apenas desenvolver habilidades cognitivas, tal como preconizado por diferentes documentos que influenciam até a hoje propostas curriculares no Brasil e no mundo, dentre eles o famoso relatório de Jacques Delors publicado em 1998.

Uma vez que pesquisas vêm desempenhando um papel cada vez mais central no desenvolvimento de Políticas Públicas como mostra Hypólito (2011), se faz amplamente necessário e urgente conhecer o que o panorama científico brasileiro apresentou na última década de pesquisas tanto no que se referem às propostas, quanto no que se referem às preocupações, achados e hipóteses para a conjuntura educacional no Brasil, visando agregar evidências de pesquisa que possam contribuir com esta construção social.

Em contrapartida ao escasso resultado de estudos que correlacionam Habilidades para Vida e Tecnologias Digitais Educacionais, foram encontrados estudos que em sua maioria investigam 
a conexão entre habilidades cognitivas e o desenvolvimento das Habilidades para Vida no contexto educacional (Ribeiro \& Fonseca, 2010; Marcelino et al., 2010; Scorsolini-Comin, 2014; Eisermann, Alice, \& Schulz, 2018), corroborando com os dados trazidos pelos autores Gomes (2008) e Schwarzbold (2011), que afirmam que estudos neste campo vêm sendo alvo de investigações científicas que focam na promoção do bem-estar social ao longo da vida. De forma que este resultado compôs a maioria dos artigos encontrados, enfatizando que as habilidades cognitivas (leitura, escrita, interpretação, etc.) correlacionam-se com Habilidades para Vida, subsidiando o desenvolvimento de habilidades complexas tais como resolução de problemas, pensamento crítico e criativo, tomada de decisão, entre outras, embasando assim o dados trazidos por Murta e colaboradores (2009) de que as Habilidades para Vida permitem a generalização da aprendizagem de habilidades para o enfrentamento saudável de possíveis situações adversas.

Desta forma, outro dado encontrado congruente com as afirmações anteriores, se refere a descrição ou referência a estudos similares feita por grande parte dos artigos encontrados, uma vez que a literatura que correlaciona Habilidades para Vida e Tecnologias Digitais Educacionais ainda está sendo ampliada, estes artigos fazem menção a outros estudos da área afins que trazem essas características, mas não os correlacionam entre si, o que mostra inclusive uma lacuna na literatura a ser preenchida por estudos futuros (Xavier, 2011).

Tabela 6: Resumo das informações dos artigos selecionados

\begin{tabular}{|c|c|c|c|c|c|}
\hline Título & Ano & HV Trabalhada & $\begin{array}{l}\text { TE } \\
\text { Utilizada }\end{array}$ & $\begin{array}{l}\text { Método } \\
\text { passível de } \\
\text { replicação }\end{array}$ & Dados Descritivos \\
\hline $\begin{array}{l}\text { A interação com jogos } \\
\text { digitais e aprimoramento do } \\
\text { controle inibitório: um } \\
\text { estudo com crianças do } \\
\text { Atendimento educacional } \\
\text { especializado }\end{array}$ & 2019 & $\begin{array}{l}\text { Controle inibitório, de } \\
\text { atenção, pensamentos e } \\
\text { de emoções }\end{array}$ & $\begin{array}{l}\text { Jogos } \\
\text { digitais }\end{array}$ & $\mathrm{Sim}$ & $\begin{array}{l}\text { Brasil, Crianças, } \\
8 \text { crianças de } 8 \text { a } \\
11 \text { anos }\end{array}$ \\
\hline $\begin{array}{l}\text { Khan Academy: tecnologia } \\
\text { favorável à aprendizagem } \\
\text { matemática }\end{array}$ & 2018 & $\begin{array}{l}\text { Pensamento crítico, } \\
\text { criativo e resolução de } \\
\text { problemas. }\end{array}$ & $\begin{array}{l}\text { Plataforma } \\
\text { virtual } \\
\text { Khan } \\
\text { Academy }\end{array}$ & Sim & $\begin{array}{l}\text { Brasil; } \\
\text { Crianças; } \\
\text { N/A }\end{array}$ \\
\hline $\begin{array}{l}\text { Letramento digital: impactos } \\
\text { das tecnologias na } \\
\text { aprendizagem da Geração Y }\end{array}$ & 2011 & Letramento digital & $\begin{array}{l}\text { Gravação } \\
\text { de vídeos }\end{array}$ & $\begin{array}{l}\text { Atende } \\
\text { parcialmente } \\
\text { o critério. }\end{array}$ & $\begin{array}{l}\text { Brasil; } \\
\text { Crianças; } \\
25 \text { crianças. }\end{array}$ \\
\hline $\begin{array}{l}\text { Can we teach to think in } \\
\text { primary schools? } \\
\text { comparative analysis of the } \\
\text { English and the Brazilian } \\
\text { National Curriculum and the } \\
\text { impact of a small-scale }\end{array}$ & 2014 & $\begin{array}{l}\text { Pensamento crítico, } \\
\text { criativo, resolução de } \\
\text { problemas } \\
\text { comunicação } \\
\text { interpessoal. }\end{array}$ & $\begin{array}{l}\text { Quadro } \\
\text { branco para } \\
\text { anotação } \\
\text { das } \\
\text { respostas }\end{array}$ & Sim & $\begin{array}{l}\text { Brasil; } \\
\text { Crianças; } \\
\text { N/A }\end{array}$ \\
\hline
\end{tabular}




\begin{tabular}{|c|c|c|c|c|c|}
\hline $\begin{array}{l}\text { cognitive enhancement study } \\
\text { in Brazil }\end{array}$ & & & & & \\
\hline $\begin{array}{l}\text { Matriz de referência para a } \\
\text { medição do alfabetismo nos } \\
\text { domínios do letramento e do } \\
\text { numeramento }\end{array}$ & 2010 & $\begin{array}{l}\text { Habilidades de } \\
\text { localização, } \\
\text { integração, elaboração, } \\
\text { avaliação, letramento e } \\
\text { numeramento }\end{array}$ & $\begin{array}{l}\text { Matriz de } \\
\text { referência. }\end{array}$ & Sim & $\begin{array}{l}\text { Brasil; } \\
\text { Crianças; } \\
\text { N/A }\end{array}$ \\
\hline $\begin{array}{l}\text { A promoção do uso de } \\
\text { estratégias cognitivas em } \\
\text { alunos do Ensino Médio }\end{array}$ & 2018 & $\begin{array}{l}\text { Organização, } \\
\text { planejamento, } \\
\text { monitoramento } \\
\text { regulação }\end{array}$ & $\begin{array}{l}\text { Ensino de } \\
\text { estratégias } \\
\text { em sala de } \\
\text { aula }\end{array}$ & Sim. & $\begin{array}{l}\text { Brasil; } \\
\text { Adolescente; } \\
26 \text { alunos de } 16 \text { a } \\
19 \text { anos. }\end{array}$ \\
\hline $\begin{array}{l}\text { Aprendizagem criativa na } \\
\text { construção de jogos digitais: } \\
\text { uma proposta educativa no } \\
\text { ensino de ciências para } \\
\text { crianças }\end{array}$ & 2018 & $\begin{array}{l}\text { Pensamento criativo, } \\
\text { letramento digital, } \\
\text { leitura e pensamento } \\
\text { crítico. }\end{array}$ & $\begin{array}{l}\text { Criação de } \\
\text { jogos } \\
\text { digitais }\end{array}$ & $\begin{array}{l}\text { Atende } \\
\text { parcialmente } \\
\text { o critério. }\end{array}$ & $\begin{array}{l}\text { Brasil; Crianças; } \\
23 \text { alunos }\end{array}$ \\
\hline $\begin{array}{l}\text { Learning strategies mediated } \\
\text { by technologies: use and } \\
\text { observation of teachers }\end{array}$ & 2018 & $\begin{array}{l}\text { Não especificado no } \\
\text { artigo }\end{array}$ & $\begin{array}{l}\text { Formulário } \\
\text { digital, }\end{array}$ & Sim & $\begin{array}{l}\text { Brasil; } \\
\text { Professores; } \\
515 \text { professores }\end{array}$ \\
\hline $\begin{array}{l}\text { Psicologia da educação e as } \\
\text { tecnologias digitais de } \\
\text { informação e comunicação }\end{array}$ & 2014 & Estudo teórico & $\begin{array}{l}\text { Estudo } \\
\text { Teórico }\end{array}$ & Não & $\begin{array}{l}\text { Brasil } \\
\text { Estudo teórico; } \\
\text { N/A }\end{array}$ \\
\hline Studying in 3D environments & 2014 & $\begin{array}{l}\text { Não especificado no } \\
\text { artigo }\end{array}$ & $\begin{array}{l}\text { Tecnologia } \\
\text { 3D }\end{array}$ & Não & $\begin{array}{l}\text { Brasil; } \\
\text { Universitários } \\
\text { N/A }\end{array}$ \\
\hline
\end{tabular}

Entretanto todos os artigos incluídos fizeram uso de diversos tipos de tecnologias educacionais e digitais, como jogos 3D, criação de programas e realidade virtual, relacionados ao desenvolvimento de Habilidades para Vida, pois como apontado anteriormente HV é construto integrador que trata de habilidades que promovem o desenvolvimento de um repertório de comportamentos adaptativos que auxiliam os indivíduos a lidarem de maneira mais positiva com as demandas da vida cotidiana, sendo este construto representado por: resolução de problemas, pensamento criativo, pensamento crítico, empatia, tomada de decisão, comunicação eficaz, relacionamento interpessoal, autoconhecimento, manejo de emoções e manejo de estresse (WHO, 1997), principalmente habilidades cognitivas (Ramos \& Garcia, 2019; Sobreira et al., 2018; Eisermann et al., 2018; Marcelino et al., 2010), apontando que o uso de tecnologias digitais educacionais é um campo que se encontra em pleno desenvolvimento. Deste modo, os dados encontrados por esta pesquisa reiteram que o campo de estudos das Habilidades para Vida no âmbito educacional é promissor ao apresentar resultados relevantes acerca de processos essenciais para êxito acadêmico do aluno, sendo fundamental destacar que essas habilidades são essenciais ao exercício pleno da cidadania, além disso, refinamentos dessas habilidades possibilitam o desenvolvimento de outras presentes no rol das $\mathrm{HV}$, tornando o indivíduo competente para manejar demandas do cotidiano (Nardi et al., 2017; Ramos et al., 2016). 
Durante o processo de análise, notou-se que os estudos encontrados conduziram experimentos que apresentaram baixo número de participantes, quando analisados de forma individual, indicando que neste campo poucas pesquisas são conduzidas em larga escala, uma vez que a população de estudantes de Educação Básica avaliada pelos estudos apresenta uma média de 11,4 anos, está majoritariamente matriculada em escolas públicas e que a média de participantes por experimento de 23,6 alunos. Porém, apesar do baixo número amostral, os estudos apresentaram grande cuidado metodológico e os achados informados eram passíveis pelas amostras selecionadas. Contudo, ressalta-se que poucos artigos mencionam ameaças à validade que pudessem afetar os resultados e a conclusão, sendo que este critério apenas foi mencionado pelos artigos que utilizaram instrumentos de medida para a coleta de dados (WHO, 2010; Paiva \& Rodrigues, 2008).

Destaca-se também que nos artigos incluídos, as HV que apareceram em maior número foram: resolução de problemas, pensamento crítico, pensamento criativo, manejo de estresse e tomada de decisão. Ademais, as habilidades cognitivas mais evidenciadas foram de desenvolvimento de leitura, escrita, letramento numérico e letramento digital. Indicando que apesar da definição da WHO (1997) para Habilidades para Vida incluir dez dimensões, as pesquisas ainda se mantêm concentradas em apenas metade das habilidades destacadas. Portanto, apesar da afirmação de Paiva e Rodrigues (2008) sobre a escola ser o ambiente ideal para o trabalho integral de desenvolvimento infantil, destaca-se nesta revisão que este espaço ainda não tem seu potencial completamente explorado e aproveitado, especialmente no que diz respeito a inserção das tecnologias digitais educacionais como ferramenta mediadora de acesso à aprendizagem e com grande potencial para o trabalho com Habilidades para Vida, o que levaria ao cumprimento da agenda educacional focada na educação integral presente em diversos documentos nacionais e mundiais (Brasil, 2018; UNESCO, 2015; WHO, 1997), de forma que este dado também se assemelha ao trabalho de Costa, Duqueviz e Pedroza (2015) e Bezarra, Lima, Brito e Santos (2019) que destacam entraves para inserir as tecnologias digitais educacionais adotadas pelos jovens no cotidiano escolar, considerando-as a favor dos processos de aprendizagem das HV (Sloan et al., 2016).

\section{Conclusão}

Este artigo apresentou uma revisão sistemática da literatura a respeito da integração das Habilidades para Vida às tecnologias digitais educacionais, fundamentada nas demandas da realidade escolar contemporânea que coloca a necessidade de o foco educacional ser para além do domínio dos conteúdos disciplinares, a favor de uma educação integral que contemple as Habilidades para Vida, e os resultados mostraram os avanços e as principais lacunas encontradas neste campo científico.

Dentre os avanços, destacaram-se os impactos positivos do uso das tecnologias digitais educacionais para o desenvolvimento de habilidades cognitivas essenciais às $\mathrm{HV}$ e o discreto aumento de produções ao longo tempo. Como lacunas, observou-se a carência de estudos que abarcassem uma discussão de cunho teórico que considerasse a importância das HV para o desenvolvimento infantil no ambiente escolar, a partir da utilização de programas educacionais mediados pelo uso de tecnologias digitais educacionais. Outra lacuna que ficou evidente foi o número reduzido de participantes nos estudos indicando um fraco potencial de generalização dos dados positivos encontrados e ausência de pesquisas conduzidas em larga escala em território nacional. Fazendo-se necessário a realização de estudos que explicitem estratégias mais claras sobre como relacionar tecnologias digitais educacionais e o desenvolvimento das HV, incluindo o descritivo dos métodos escolhidos que foram responsáveis por viabilizar tais relações, assim de 
fato ampliando as possibilidades de tais estudos virem a fomentar políticas públicas educacionais mais adaptadas à esta era tecnológica.

Por fim, este trabalho revelou que muito ainda precisa ser feito para que ocorra um investimento em pesquisas a favor do desenvolvimento das tecnologias digitais educacionais aplicadas à disseminação das Habilidades de Vida no contexto escolar, contribuindo de modo efetivo para a formação de alunos capazes de lidar com as diversas adversidades encontradas em ambientes sociais, profissionais e pessoais.

\section{Referências}

Bastos, C. A. R., \& Siqueira, W. M. (2020). Repensando o Ensino com Novas Tecnologias, Design Thinking e Experiência do aluno: Um Estudo Qualitativo com base em Formação Docente. Revista Brasileira de Informática $\mathrm{Na}$ Educação, 573-595. doi: $\underline{10.5753 / R B I E .2020 .28 .0 .573}$ [GS Search]

Bezarra, M. M., Lima, E. C., Brito, F. W. C., \& Santos, A. C. B. dos. (2019). Geração Z: Relações de uma Geração Hipertecnológica e o Mundo do Trabalho. Revista Gestão Em Análise, 8(1), 136-149. doi: 10.12662/2359-618xregea.v8i1.p136-149.2019 [GS Search]

Brasil. (2018). Base Nacional Comum Curricular - Educação é a base. 1-472. Disponível em http://basenacionalcomum.mec.gov.br/images/BNCC_EI_EF_110518_versaofinal_site. pdf

Catania, A. C. Aprendizagem: Linguagem, comportamento e cognição. Porto Alegre: Artmed, 1999. [GS Search]

Costa, S. R. S., Duqueviz, B. C., \& Pedroza, R. L. S. (2015). Tecnologias Digitais como instrumentos mediadores da aprendizagem dos nativos digitais. Revista Quadrimestral Da Associação Brasileira de Psicologia Escolar e Educacional, SP, 19(3), 603-610. doi: $\underline{10.1590 / 2175-3539 / 2015 / 0193912}$ [GS Search]

Delors, J., Al-Mufti, I., Amagi, I., Carneiro, R., Chung, F., Geremek, B., ... Nanzhao, Z. (1998). Educação um Tesouro a Descobrir - Relatório para a UNESCO da Comissão Internacional sobre Educação para o século XXI. Lisboa. [GS Search]

Eisermann, J. I., Alice, J., \& Schulz, T. (2018). Khan Academy: tecnologia favorável à aprendizagem matemática. Ensino de Matemática Em Debate, 5(2), 186-200. [GS Search]

Gomes, M. C. D. S. (2008). Letramento e inclusão social (pp. 65-67). pp. 65-67. Buenos Aires: XV Jornadas de Investigación y Cuarto Encuentro de Investigadores en Psicología del Mercosur. [GS Search]

Gorayeb, R. (2002). O ensino de habilidades de vida em escolas no Brasil. Psicologia, Saúde \& Doenças, 3(2), 213-217. [GS Search]

Hypólito, Á. (2011). Políticas públicas e educação. Revista Retratos Da Escola, 175-177.

Marcelino, R., Silva, J. B. da, Gruber, V., Bilessimo1, S., Oliveira1, J., Santana1, S., \& Mello, I. (2010). Studying in 3D Environments. International Journal of Emerging Technologies in Learning, 5(4), 1-10. doi: 10.3991/ijoe.v10i3.3340 [GS Search]

Marques, S. (2014). Can we Teach to Think in Primary Schools? A Comparative Analysis of the English and the Brazilian National Curriculum and the Impact of a Small-scale Cognitive Enhancement Study in Brazil. Procedia - Social and Behavioral Sciences, 137, 138-146. doi: 10.1016/j.sbspro.2014.05.266 [GS Search] 
Minto, E. C., Pedro, C. P., Netto, J. R. da C., Bugliani, M. A. P., \& Gorayeb, R. (2006). Ensino de habilidades de vida na escola: uma experiência com adolescentes. Psicologia Em Estudo, 11(3), 561-568. doi: 10.1590/S1413-73722006000300012 [GS Search]

Morales, M. R., Benitez, M. H., \& Santos, D. A. (2013). Habilidades para la vida (cognitivas y sociales) en adolescentes de una zona rural. Revista Electrónica de Investigación Educativa, 15(3), 98-113. [GS Search]

Murta, S. G., Borges, F. A., Cruvinel, D., Eliana, R., Rocha, P., \& Lopes De Menezes, J. C. (2009). Prevenção primária em saúde na adolescência: avaliação de um programa de habilidades de vida (Vol. 14). doi: 10.1590/S1413-294X2009000300001 [GS Search]

Nardi, P. C., Ferreira, I. M. F., \& Neufeld, C. B. (2017). Resultados preliminares do Programa PRHAVIDA em crianças com escores clínicos de ansiedade, depressão e estresse. Contextos Clínicos, 10(1), 74-84. doi: 10.4013/ctc.2017.101.06 [GS Search]

Neufeld, C. B., Daolio, C. C., Cassiano, M., Rossetto, C. P. F., \& Cavenage, C. C. (2014). Phavida - Um Programa Cognitivo-Comportamental de Habilidades de Vida Para Crianças e Adolescentes. In Intervenções e Pesquisas em Terapia Cognitivo-Comportamental com Indivíduos e Grupos (pp. 80-116). [GS Search]

Paiva, F. S. de, \& Rodrigues, M. C. (2008). Life Skills: A preventive strategy against the consumption of psychoactive substances in the school context. Estudos e Pesquisas Em Psicologia, 8(3), 672-684. [GS Search]

Ramos, D. K., \& Garcia, F. A. (2019). Digital games and improvement of the inhibitory control: A study with children in specialized educational service. Revista Brasileira de Educacao Especial, 25(1), 37-54. doi: 10.1590/S1413-65382519000100003 [GS Search]

Ramos, D. K., Lorenset, C. C., \& Petri, G. (2016). Jogos Educacionais: Contribuições da Neurociência à Aprendizagem. Revista X, 2(1), 1-17. doi: $\underline{10.5380 / r v x . v 2 i 1.2016}$ [GS Search]

Ribeiro, V. M., \& Fonseca, M. C. (2010). Matriz de referência para a medição do alfabetismo nos domínios do letramento e do numeramento. Estudos Em Avaliação Educacional, 21(45), 147. doi: $\underline{10.18222 / \text { eae214520102031 [GS Search] }}$

Sargiani, R. de A., \& Maluf, R. M. (2018). Linguagem, Cognição e Educação Infantil: Contribuições da Psicologia Cognitiva e das Neurociências Lenguaje, Cognición y Educación infantil: Contribuciones de la Psicología Cognitiva y de las Neurociencias. Psicologia Escolar e Educacional, 22(3), 477-484. doi: 10.1590/2175-35392018033777 [GS Search]

Schwarzbold, C. (2011). Desenvolver a competência leitora: desafio ao professor do ensino fundamental. Universidade Federal de Pelotas. [GS Search]

Scorsolini-Comin, F. (2014). Psicologia da educação e as tecnologias digitais de informação e comunicação. Psicologia Escolar e Educacional, 18(3), 447-455. doi: 10.1590/2175$\underline{3539 / 2014 / 0183766}$ [GS Search]

Sloan, S., Gildea, A., Miller, S., Poulton, L., Egar, C., \& Thurston, A. (2016). Evaluation of Zippy's Friends for improving socio-emotional and academic outcomes in six to seven year olds: Protocol for a cluster randomised controlled trial and process evaluation. International Journal of Educational Research, 82(2016), 200-209. doi: 10.1016/j.ijer.2017.01.003 [GS $\underline{\text { Search }] ~}$

Sobreira, E. S. R., Viveiro, A. A., \& d'Abreu, J. V. V. (2018). Aprendizagem criativa na construção de jogos digitais: uma proposta educativa no ensino de ciências para crianças. Tecné Episteme y Didaxis: TED, (44). doi: 10.17227/ted.num44-8990 [GS Search] 
WHO. (1997). Life skills education for children and adolescents in schools. Retrieved from https://www.scielo.br/pdf/epsic/v14n3/a01v14n3.pdf. [GS Search]

WHO. (2004). The World Health Report. Geneve.

WHO. (2010). AS REDES DE ATENÇÃO À SAÚDE.

Xavier, A. C. (2011). Digital literacy: Impacts of technology on learning from Generation Y. Calidoscopio, 9(1), 3-14. doi: 10.4013/cld.2011.91.01 [GS Search] 